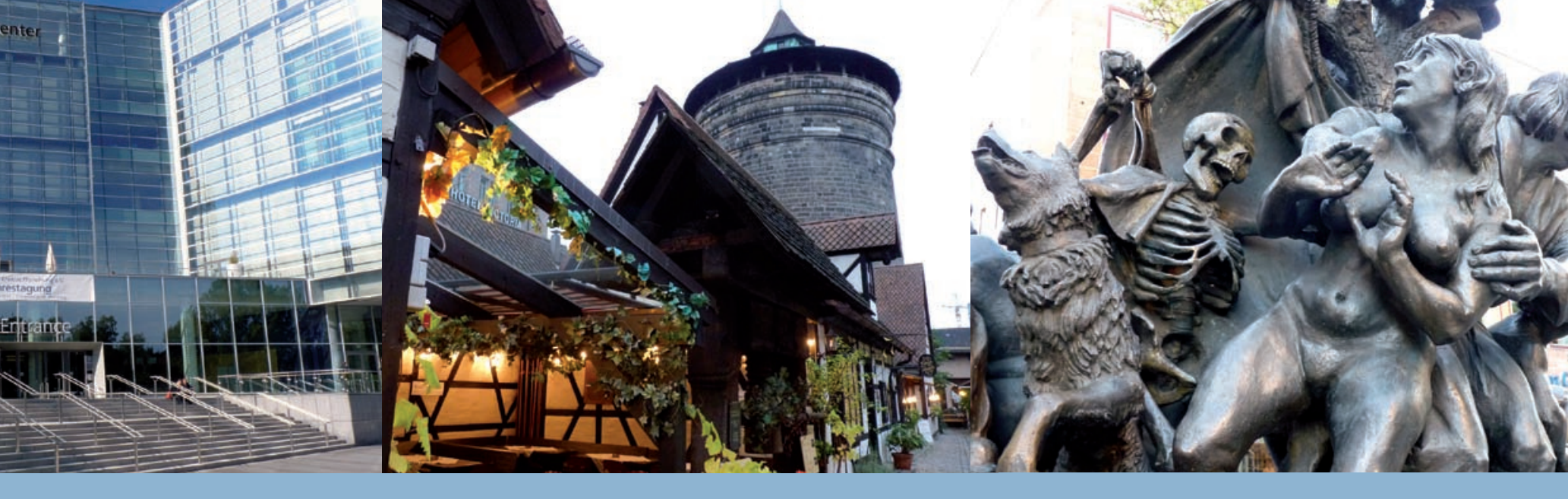

\section{Entwicklung der Kosten für Herzkrankheiten}

In der Langzeitbeobachtung fällt eine rasante Kostensteigerung auf: Von 2002 bis 2008 stiegen die Krankheitskosten insgesamt um 35512 Milliarden Euro (Männer 20259 , Frauen 15253) an. Dies bedeutet einen Anstieg von 16,2\% (Männer + 22,5\%, Frauen $+11,8 \%)$. Für die ischämischen Herzkrankheiten lag die Zuwachsrate der Krankheitskosten von 2002 bis 2008 bei den
Männern bei 1,6\%. Bei den Frauen ergab sich sogar ein Rückgang von 13,5\%. Demgegenüber betrug die Zuwachsrate für den akuten Myokardinfarkt bei den Männern $82,3 \%$ und bei den Frauen $62,2 \%$. Bei der Herzinsuffizienz lag die Zuwachsrate der Männer mit 56,6\% doppelt so hoch wie die der Frauen mit 23,5\%.

Ein vergleichbares Bild bietet sich für die angeborenen Fehlbildungen $(+69,5 \%$ bei den Männern, $+32,2 \%$ bei den Frauen). Bei den vier ausgewählten Herzkrankheiten entfiel der mit Abstand größte Anteil der Krankheitskosten auf die Krankenhäuser. Bei den ischämischen Herzkrankheiten waren es 52\% (Männer 56\%, Frauen 44,7\%), beim akuten Myokardinfarkt 71\% (Männer $71,3 \%$, Frauen $70,3 \%$ ), bei der Herzinsuffizienz 46,8\% (Männer 55,8\%, Frauen 40,2\%) und bei den angeborenen Fehlbildungen des Kreislaufsystems 72,4\% (Männer 73,6\%, Frauen $71,1 \%$ ).

\title{
Der Nürnberger Trichter: kardiologische Neuheiten im Schnelldurchlauf
}

Die Assoziation mit dem „Nürnberger Trichter“ lag nahe: 599 Vorträge in zwei Tagen wurden den Teilnehmern des Doppelkongresses der DGK Mitte Oktober im Nürnberger Kongresszentrum angeboten, um nicht zu sagen „eingetrichtert“. Die beiden Kongresspräsidenten sorgten für ein aktuelles, teilweise brisantes Programm: DGK-Tagungspräsident Prof. Udo Sechtem, Stuttgart, sprach in seiner Eröffnungsrede die zukünftigen Probleme der Kardiologieweiterbildung an und Prof. Andreas Schuchert, Neumünster, Präsident der angekoppelten Jahrestagung der Arbeitsgruppe Schrittmacher und Arrhythmie, war besonders stolz auf die Life-CaseÜbertragungen aus dem Bereich der gesamten Kardiologie, drei davon speziell aus der Rhythmologie (s. Interview S. 14). Der Vorsitzende der DGK, Prof. Michael Böhm aus Homburg/Saar, hatte in der Eröffnungsveranstaltung die traurige Pflicht, den Nestor der deutschen Kardiologie, Prof. Franz Loogen, zu würdigen; dieser war am 3. September im Alter von 91 Jahren verstorben. Loogen hatte in Düsseldorf den ersten kardiologischen Lehrstuhl Deutschlands ins Leben geru-

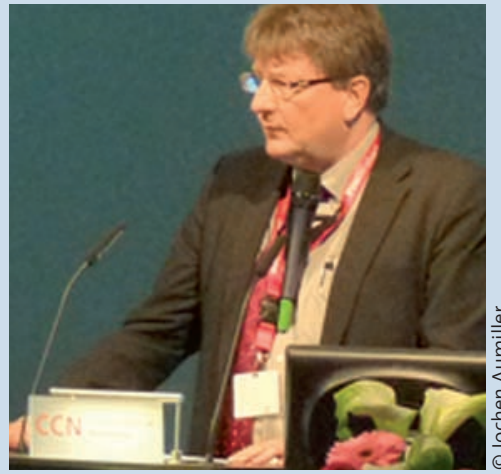

Der DGK-Vorsitzende Prof. Michael Böhm

fen - gegen den Widerstand der Inneren Medizin, die eine Spaltung des Fachs verhindern wollte.

\section{Nachwuchsförderung}

Künftig sollen nicht nur promovierte Studenten zur DGK-Jahrestagung eingeladen werden, sondern bereits in den Leistungskursen der Schulen auf die Möglichkeiten der Kardiologie aufmerksam gemacht werden. Erklärtes Ziel der DGK ist es, die jungen Kollegen in ihrer wissenschaftlichen Qualifikationsphase zu unterstützen, auch mit
Drittmitteln, die derzeit nicht ausgeschöpft werden. Für Assistenten soll auf der nächsten Jahrestagung ein eigener Seminarblock gestaltet werden, in dem wissenschaftliche Methoden im Mittelpunkt stehen.

Unklar blieb, wie die intensivierte Nachwuchsförderung mit dem sich abzeichnenden Kardiologenüberschuss in Einklang gebracht werden kann.

\section{Herzforschungszentrum im Plan}

Zum geplanten Deutschen Herzforschungszentrum konnte Böhm melden, dass bislang alles nach Plan verlaufe. 22 Anträge werden derzeit von einem internationalen Expertengremium geprüft. Anfang November ist ein erstes Hearing mit den Antragstellern geplant; in einer Vorauswahl werden dann zehn Zentren benannt. Die logistischen Zügel sollen in einem Nationalen Institut für Kreislauferkrankungen in Berlin zusammenlaufen. Was in jedem Fall erreicht wird: die bessere Vernetzung der deutschen Kardiologie - bislang eher ein Defizitmodell. Also alles im grünen Bereich, meinte Böhm.

(J.A.) II 